\title{
Hierarchy of minimal flow units in the logarithmic layer
}

\author{
Oscar Flores $^{\mathrm{a})}$ and Javier Jiménez ${ }^{\mathrm{b})}$ \\ School of Aeronautics, Universidad Politécnica de Madrid, 28040 Madrid, Spain
}

(Received 26 April 2010; accepted 21 June 2010; published online 28 July 2010)

\begin{abstract}
The minimal simulation boxes of the buffer layer of turbulent channels can be extended to the logarithmic and outer regions, where they contain a segment of streamwise velocity streak, and a vortex cluster. Smaller boxes restrict "healthy" turbulence closer to the wall, to a layer whose thickness scales with the spanwise size of the box. These minimal boxes burst quasiperiodically, and the bursting period for a band of wall distances grows linearly away from the wall, independently of the box size within the limits within which turbulence is well represented. (C) 2010 American Institute of Physics. [doi:10.1063/1.3464157]
\end{abstract}

The observation that the buffer and viscous layers of wall bounded flows can be simulated in periodic boxes of "minimal" dimensions ${ }^{1}$ has been useful in understanding wall turbulence because it allows individual flow features to be studied in relative isolation from their mutual interactions. For example, it has led to the demonstration that the "bursts" observed in experiments ${ }^{2}$ are, at least in part, temporal modulations of flow structures rather than spurious effects of the passage of steady features near the experimental probes. ${ }^{3}$ In this letter, we show that similar minimal boxes exist for the logarithmic and outer layers of turbulent channels, and that they also burst. A preliminary report of those results is Ref. 4.

The simulations are turbulent channels of half-height $h$, using the same spectral code as in Ref. 5, in boxes extending from wall to wall, but smaller than those required to accommodate the turbulent scales of the outer region. The streamwise, wall-normal and spanwise directions are denoted by $x$, $y$, and $z$, respectively, and the corresponding velocity components by $u, v$, and $w$. Global averages, defined over wallparallel planes and time, are denoted with capital letters. Primes represent root-mean-squared fluctuation intensities with respect to these global averages. Wall units are defined in terms of the kinematic viscosity and of the friction velocity $u_{\tau}$, and denoted by a + superindex. The parameters of the main simulations are listed in Table I.

The mean velocity profiles of the small-box simulations are compared in Fig. 1 to those of full-box turbulent channels at similar Reynolds numbers. ${ }^{6,7}$ As in the minimal channels of the buffer layer, ${ }^{1}$ the profiles of the small boxes are characterized by large parabolic outer components, because the length scale beyond a certain $y$ is fixed by the box, instead of by the distance to the wall. ${ }^{8}$ However, these flows are not minimal in the same sense of those in the buffer layer. Choosing a smaller box does not lead to the decay of turbulence, but to a reduction of the layer with "healthy" turbulence, where the flow statistics are in agreement with full-

\footnotetext{
${ }^{a)}$ Present address: Mechanical Engineering, U. of Washington, Seattle, WA 98195, USA.

b) Also at Centre for Turbulence Research, Stanford University, Stanford, CA 94305, USA.
}

box turbulent channels (see discussion of Fig. 2 below). In that sense, the boxes in Ref. 1 are the innermost members of a hierarchy in which progressively smaller wall-attached structures are isolated by progressively smaller numerical boxes. The special feature of the minimal boxes of the buffer layer is that they cannot be restricted any further, while larger boxes isolate more complicated, fully multiscale structures, that reach from the wall farther into the core flow.

Figure 1(a) displays the deviations from the nominal mean velocity profile of three small-box channels. The smallest box corresponds to the minimal buffer-layer dimensions, and its profile diverges just above the buffer layer. The other two, with larger boxes, reproduce well the logarithmic layer over taller regions. If we arbitrarily define the limit of healthy turbulence as the location $y_{d}$ of the minimum in the velocity excess, it turns out to be roughly proportional to the box size. The critical dimension appears to be the spanwise periodicity, with $y_{d} \approx 0.3 L_{z}$. That is seen in Fig. 1(b), which includes the three cases in Fig. 1(a), plus other combinations in which one of the box dimensions was increased or decreased with respect to the aspect ratio $L_{x} / L_{z}=2$ of the other two log-layer boxes. Doubling the box length, as in case NL1900, does not improve the profile, while shortening it to $L_{x} / L_{z}=1 / 2$ degrades it moderately. The dependence with $L_{z}$ agrees with earlier experiments ${ }^{9}$ that reproduced a healthy logarithmic profile in a wide numerical box whose streamwise dimension was of the order of the buffer layer minimal limit $\left(L_{x}^{+}=380 \approx h^{+}\right)$. The proportionality constant between $y_{d}$ and $L_{z}$ is consistent with $L_{z}$ being the limiting factor to the largest self-similar attached vortex cluster ${ }^{10,11}$ resolved in the simulation. These clusters have aspect ratios 3:1:1.5, and they are associated in average with a wall-normal velocity ejection. ${ }^{10}$ Therefore, it makes sense that a minimal box for a given height $\left(y_{d}\right)$ should at least be wide enough to contain an ejection $(v>0)$ and its associated sweep $(v<0)$, yielding $L_{z} \approx 2 \times 1.5 y_{d}$. Note that extending this estimate to the center of the channel would imply that the smallest box that can be considered a full numerical channel should be of the order of $L_{z} \approx 3 h$.

The spectra of the streamwise and wall-normal velocities in the logarithmic layer are given in Fig. 2(a), together with the box dimensions $L_{x} / y=6$ and $L_{z} / y=3$ suggested by Fig. 
TABLE I. Parameters of the main simulations. $L_{x}$ and $L_{z}$ are the streamwise and spanwise box periodicities. Case names are as follows: minimal (M), narrow $(\mathrm{N})$, and wide $(\mathrm{W})$.

\begin{tabular}{lrrr}
\hline \hline Case & $h^{+}$ & $L_{x}^{+}$ & $L_{z}^{+}$ \\
\hline M180 & 181 & 455 & 125 \\
M1900 & 1690 & 440 & 110 \\
N1900 & 1710 & 1340 & 670 \\
NL1900 & 1730 & 2710 & 680 \\
W1900 & 1840 & 2900 & 1450 \\
WS1900 & 1835 & 770 & 1440 \\
\hline \hline
\end{tabular}

1(b). It shows that the minimal boxes are large enough to contain a substantial fraction of the wall-normal energy, but only a small part of the $u$-streaks. Figure 2(b) corroborates this, showing that the profiles for the wall-normal velocity intensities are almost the same in the minimal and full computational boxes for $y<y_{d}$. The streamwise and spanwise (not shown) fluctuations are lower in the minimal boxes than in the full channel, and they are progressively damped as the
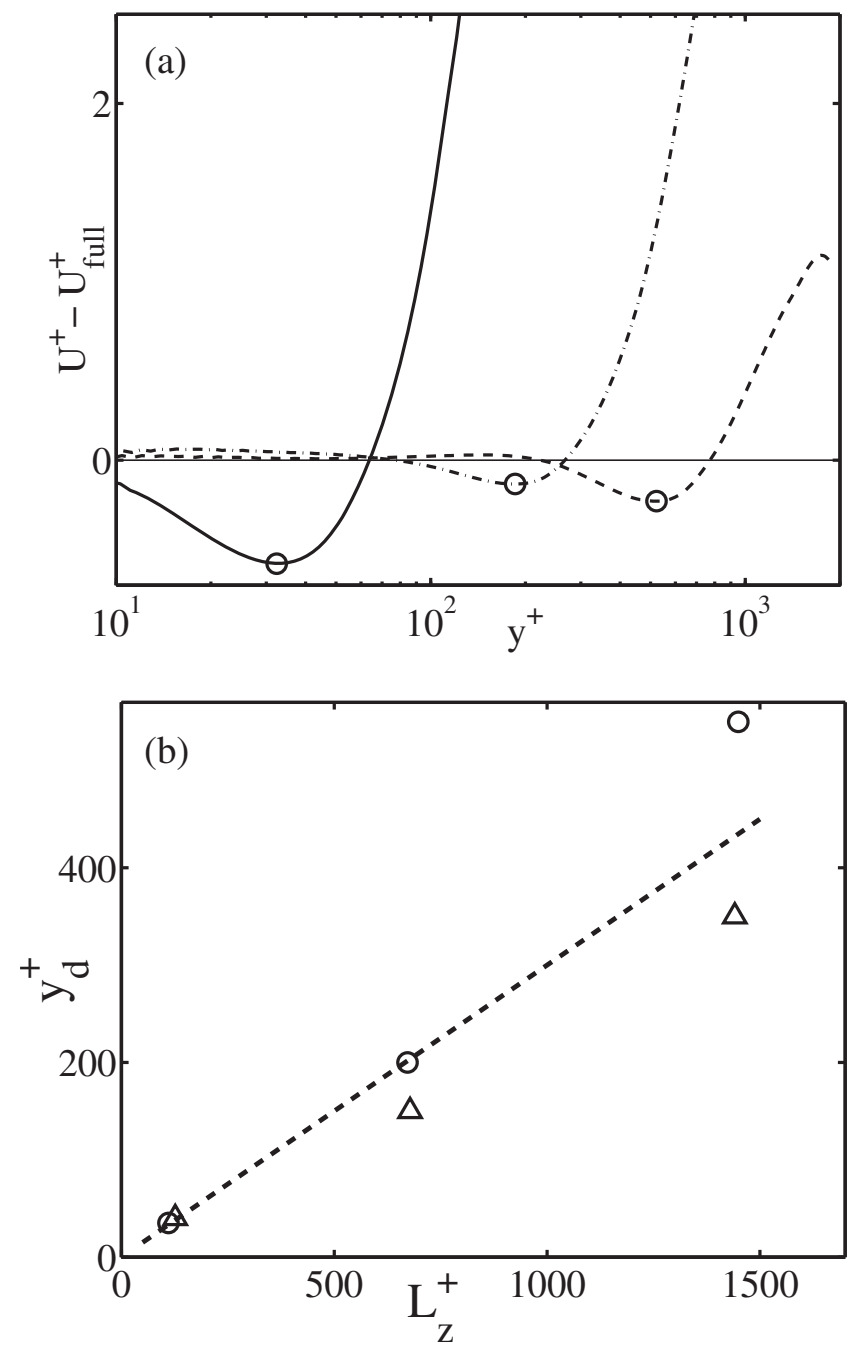

FIG. 1. (a) Excess of the small-box mean velocity profiles over a full channel (Ref. 7). —, M1900; - - -, N1900; and -- -, W1900. (b) Height of the small-box logarithmic profiles as a function of the spanwise box size. $\bigcirc$, as in (a); $\triangle$, M180, ML1900, and WS1900; - - , $y_{d}=0.3 L_{z}$.
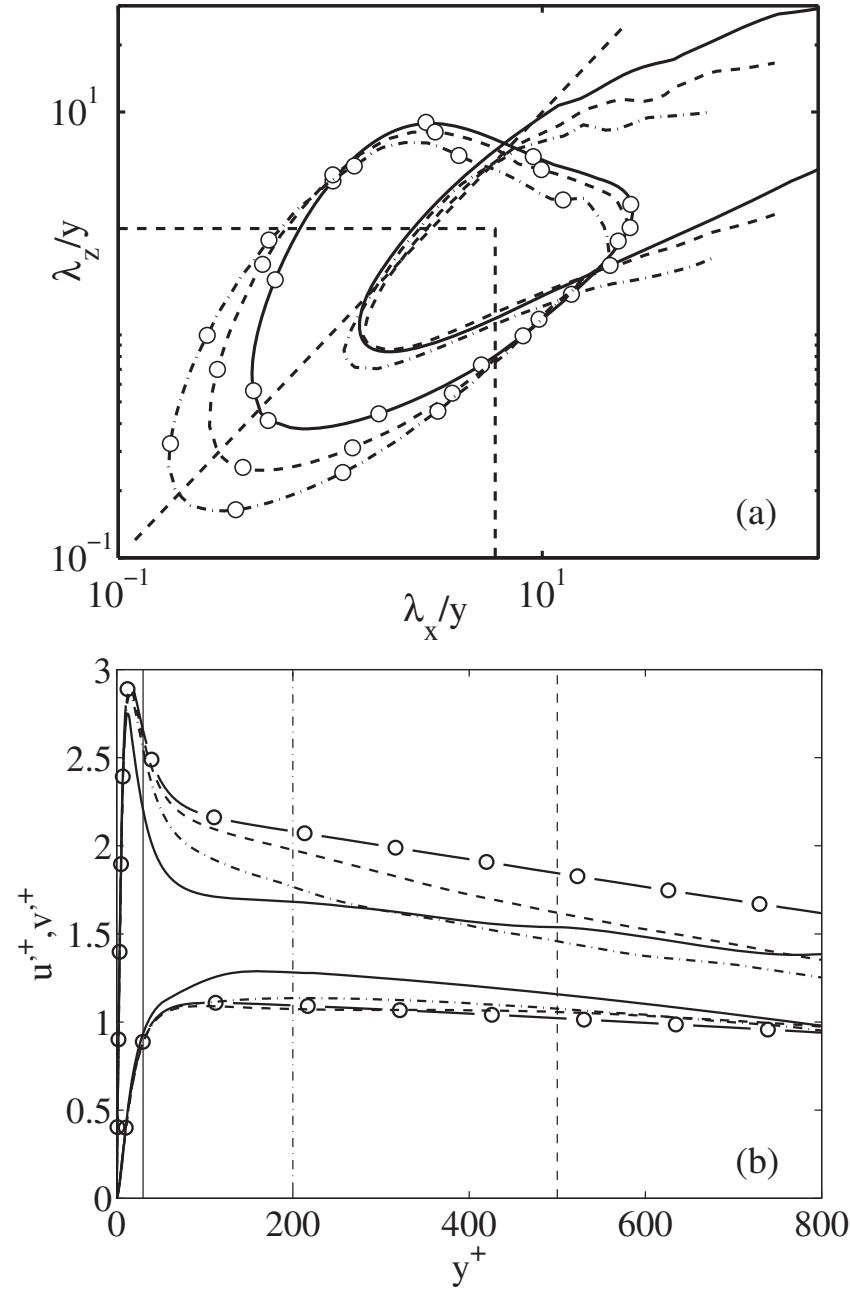

FIG. 2. (a) Premultiplied spectra of $u$ (without symbols) and $v$ (with symbols) in terms of the wavelengths scaled with $y$. Channel at $h^{+}=2000$. - , $y / h=0.1 ;---, 0.2$; and $-\cdots, 0.4$. The dashed rectangle is $\left(\lambda_{x}, \lambda_{z}\right)$ $=(6,3) y$, and the diagonal is $\lambda_{x}=\lambda_{z}$. Isolines are 0.3 times the maximum of each spectrum. (b) Vertical profiles of $u^{\prime}$ and $v^{\prime}$. $\bigcirc$, full channel at $h^{+}$ $=2000 ;-, \mathrm{M} 1900 ;-\cdot-, \mathrm{N} 1900$; and,$--- \mathrm{W} 1900$. The vertical lines are $y^{+}=y_{d}^{+}$

box is reduced. On the other hand, it was shown in Ref. 5 that the energy spectra of small computational boxes agree well with those of larger ones up to their smallest nonzero numerical wavenumbers, and the same is true in these cases.

Minimal boxes burst in time, as shown by the two $y-t$ diagrams in Fig. 3 of a short segment of the history of the Reynolds stress, $-\langle u v\rangle$, of W1900, integrated over wallparallel planes. The stresses in the top panel have been averaged only over ejections $(u<U, v>0)$, and those of the bottom panel over sweeps $(u>U, v<0)$. They are modulated intermittently in time, and it is interesting that the bursting events do not appear to originate either from the wall or from the outside. Ejections rise and sweeps descend at approximately the same time, as if being driven by an unseen common event filling most of the lower half of the computational box. In both cases, the vertical advection velocity is close to $u_{\tau}$. Figure 3 also displays isosurfaces of the streamwise velocity at two moments in which the flow is respectively quiescent [Fig. 3(b)] and bursting [Fig. 3(c)]. The raised ridges mark the low-velocity streaks. During the 

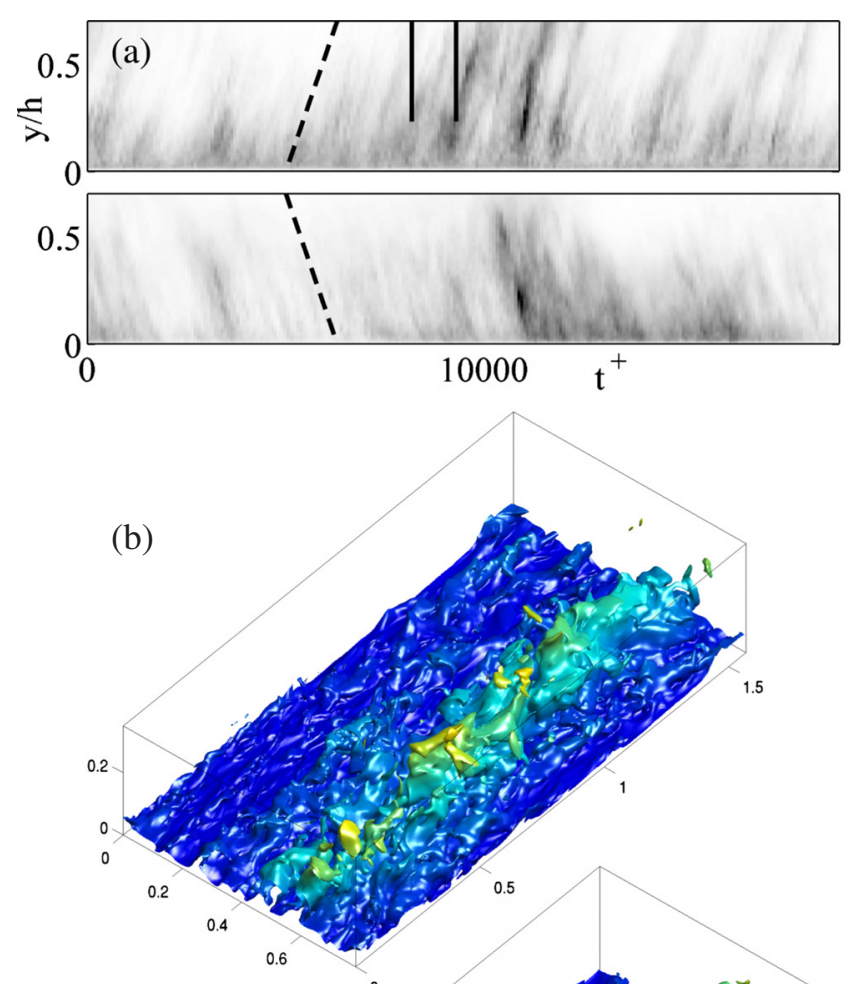

(c)

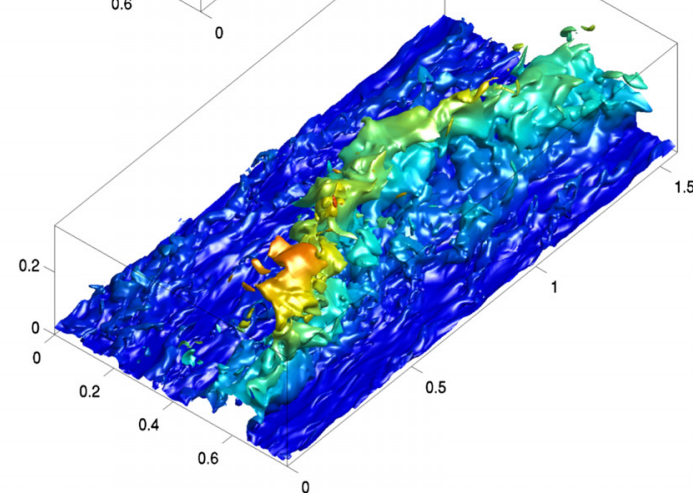

FIG. 3. (Color online) (a) Temporal evolution of the Reynolds stress $-\langle u v\rangle$ averaged of wall-parallel planes in W1900. Dark shades correspond to higher stress. In the top panel, $\langle u v\rangle$ is only averaged for ejections $(u$ $<0, v>0)$, and in the bottom one, only for sweeps $(u>0, v<0)$. The inclined dashed lines are $d y / d t= \pm u_{\tau}$. [(b) and (c)] Isosurfaces $u^{+}=15$ at the moments marked by the two vertical lines in (a) shaded with the distance from the wall. Dark shades (blue online) are close to the wall, lighter shades (orange online) correspond to $y / h \approx 0.35$. The flow is from bottom-left to top-right.

burst, the streak becomes wavy and gets eventually disorganized before reforming for the next burst. The effect on the streamwise velocity is to bring down high-speed mean velocity toward the wall, no doubt because the low-velocity streak is destroyed. The process is strongly reminiscent of the one described in Ref. 1 for the bursts in the buffer layer, but the scale is about 20 times larger, and inspection of the isosurfaces reveals that they are irregular and turbulent themselves.

Figure 4 displays the joint probability density function of the streamwise and wall-normal energies in a minimal box, integrated over its healthy layer, and compares it with the same quantities computed over similar sub-boxes of a full channel. If we consider the streamwise energy as a surrogate for the intensity of the streak, and the wall-normal one as the

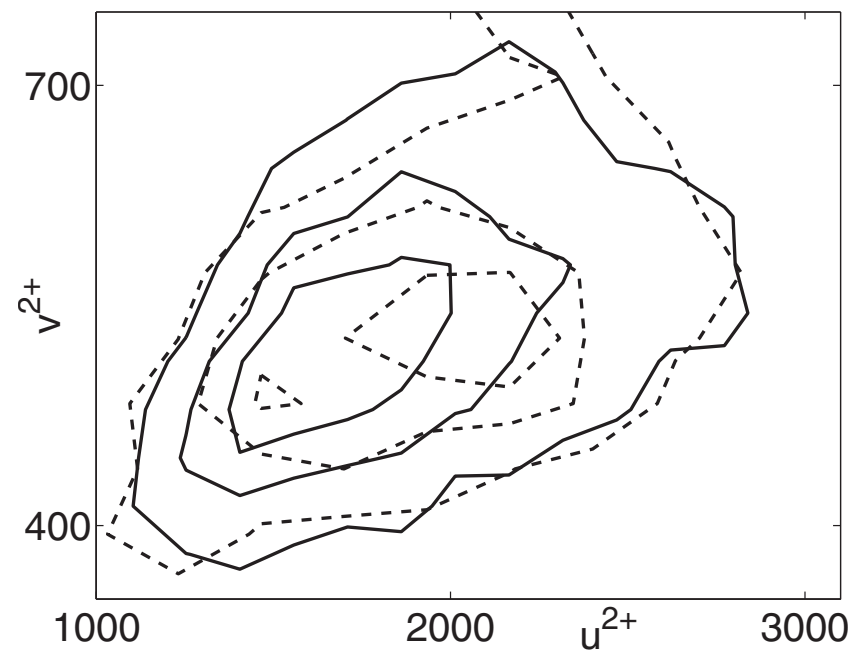

FIG. 4. The dashed contours are the probability density function of $u^{\prime 2}$ and $v^{\prime 2}$ in W1900, integrated over its whole box below $y^{+}=500$. The solid contours are the same quantities in a full channel (Ref. 7) with $h^{+}=2000$, computed over sub-boxes of the same size. Isolines contain $0.3,0.6$, and 0.9 of the total probability mass.

intensity of the ejection, the figure traces the interplay of the two components during the regeneration cycle. In the minimal boxes, the flow traverses its probability distribution counterclockwise, as for a similar representation of the buffer layer. ${ }^{3}$ Starting at the quiescent part of the cycle (low $u^{\prime}$ and $v^{\prime}$ ), the streak grows first during a stage in which turbulent energy production is larger than turbulent dissipation. As the streak becomes more energetic, it generates a wall-normal burst that enhances turbulent dissipation and eventually destroys the streak. Although it is not possible to follow the time evolution of the fluctuations in the full channel, the fact that both flows share the same region of the parameter space suggests that they undergo similar bursting processes.

The bursting period is not constant, and depends both on the simulation box and on the distance to the wall. Figure 5(a) displays frequency spectra of the time histories of the difference between the production and the dissipation, integrated over bands of wall distance, which move to shorter periods as the bands move closer to the wall. The corresponding periods are estimated in Fig. 5(b) as the centers of gravity of the semilogarithmic plots in Fig. 5(a), as in Ref. 3. All boxes approximately follow $T_{c}=6 y / u_{\tau}$, but the narrower ones peel off from the general trend at about the same height at which their mean profiles deteriorate. Note that, for bands in the buffer layer $\left(y^{+}=0-50\right)$, the period determined in this way is $T^{+} \approx 250$, somewhat shorter than the one found in Ref. 3 by analyzing the evolution of the integrated friction coefficient. Different quantities participate differently from the various layers of the flow, and may produce slightly different results. The values in Fig. 5(b) can therefore only be considered as indicative. For example, the two solid symbols in Fig. 5(b) were obtained by counting directly the number of times that the orbit in a diagram similar to Fig. 4, but drawn in terms of the energy production and dissipation, ${ }^{12}$ circles the center of gravity of its probability distribution. The results are consistent with the general trend of the periods ob- 

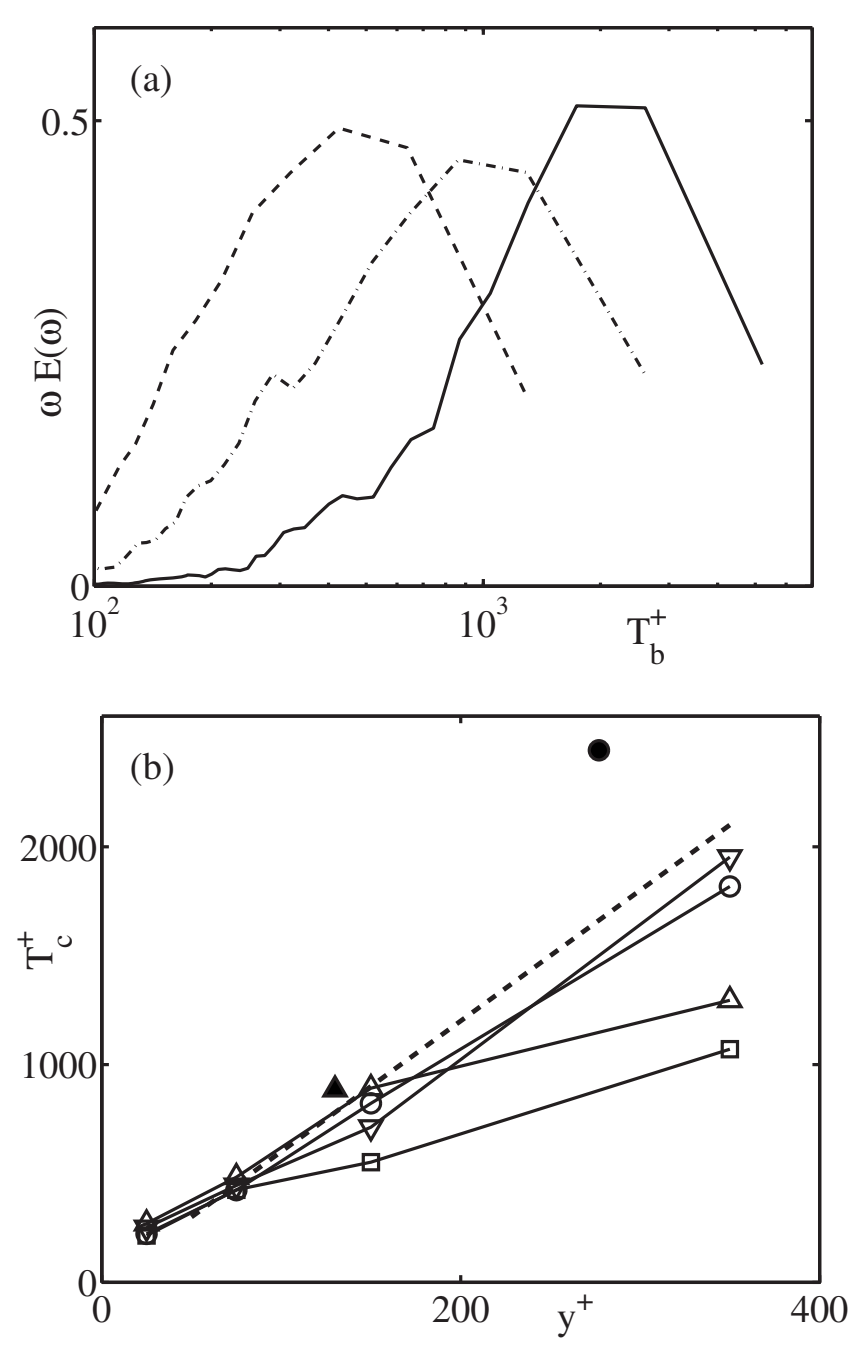

FIG. 5. (a) Premultiplied frequency spectra of the energy production minus dissipation in case W1900, integrated over the wall-parallel planes and bands of $y$, as a function of the period, $T=2 \pi / \omega .---, y^{+}=50-100 ;-\cdot-$, 100-200; and -, 200-500. (b) Bursting period, estimated for the different small boxes, and expressed as function of the center of the averaging $y$-band. O, W1900; $\triangle$, N1900; $\nabla$, WS1900; and $\square$, NL1900. The solid symbols are estimated directly from the production-dissipation orbits of the corresponding channels. The dashed diagonal is $T_{c}=6 y / u_{\tau}$.

tained from the frequency spectra, although somewhat higher.

In summary, we have shown that the flow in the logarithmic layer of turbulent channels can be restricted to an array of identical minimal boxes, in the same way as that in the buffer layer. The boxes typically contain a short stretch of a streak of the streamwise velocity, and an attached vortex cluster, ${ }^{10,11}$ but are much larger than the minimal boxes of the buffer layer, and the structures that they contain are themselves turbulent. Smaller boxes do not result in the decay of the turbulence, but in its restriction to a thinner layer near the wall. The flow in the minimal boxes undergoes quasiperiodic bursts, during which the streak becomes wavy, dis- sipation increases, and the streak is eventually destroyed. The range of properties traversed by the minimal flow during the bursting event agrees well with the statistics compiled over sub-boxes of similar sizes in full simulations, suggesting that the minimal dynamics are also part of the full flow. Both the height of the layer with healthy turbulence and the bursting period increase linearly with the spanwise dimension of the box.

It is known that destroying the dynamics of the buffer layer has essentially no influence over the statistics of the logarithmic layer, or over the properties of its attached vortex clusters, ${ }^{11,13}$ and the present results show that well-developed outer large-scale structures are also unnecessary. In fact, the $y$ - $t$ diagrams in Fig. 3 do not provide any evidence that the bursts originate either from the wall or from the center of the channel. What the present observations suggest is that, although there is no doubt that there are interactions among structures of different sizes, and from different heights within the channel, the primary generation mechanism for structures of a given size must be sought in processes of roughly the same dimensions.

This work was supported by the CYCIT Grant No. TRA2009-11498. O.F. was partially supported by a fellowship from the Spanish Ministry of Education and Science.

${ }^{1}$ J. Jiménez and P. Moin, "The minimal flow unit in near-wall turbulence," J. Fluid Mech. 225, 213 (1991).

${ }^{2}$ H. T. Kim, S. J. Kline, and W. C. Reynolds, "The production of turbulence near a smooth wall in a turbulent boundary layer," J. Fluid Mech. 50, 133 (1971).

${ }^{3}$ J. Jiménez, G. Kawahara, M. P. Simens, M. Nagata, and M. Shiba, "Characterization of near-wall turbulence in terms of equilibrium and 'bursting' solutions," Phys. Fluids 17, 015105 (2005).

${ }^{4}$ O. Flores and J. Jiménez, "The minimal logarithmic region," Bulletin of the American Physical Society, 60th Meeting of the DFD/APS, 2007, Salt Lake City (Am. Phys. Soc., 2007), pp. AE-04.

${ }^{5}$ J. C. del Álamo, J. Jiménez, P. Zandonade, and R. D. Moser, "Scaling of the energy spectra of turbulent channels," J. Fluid Mech. 500, 135 (2004).

${ }^{6}$ J. Kim, P. Moin, and R. D. Moser, "Turbulence statistics in fully developed channel flow at low Reynolds number," J. Fluid Mech. 177, 133 (1987).

${ }^{7}$ S. Hoyas and J. Jiménez, "Scaling of the velocity fluctuations in turbulent channels up to $\mathrm{Re}_{\tau}=2003$," Phys. Fluids 18, 011702 (2006).

${ }^{8}$ J. Jiménez and A. Pinelli, "The autonomous cycle of near wall turbulence," J. Fluid Mech. 389, 335 (1999).

${ }^{9} \mathrm{~S}$. Toh and T. Itano, "Interaction between a large-scale structure and nearwall structures in channel flow," J. Fluid Mech. 524, 249 (2005).

${ }^{10}$ J. C. del Álamo, J. Jiménez, P. Zandonade, and R. D. Moser, "Self-similar vortex clusters in the turbulent logarithmic region,” J. Fluid Mech. 561, 329 (2006).

${ }^{11}$ O. Flores, J. Jiménez, and J. C. del Álamo, "Vorticity organization in the outer layer of turbulent channels with disturbed walls," J. Fluid Mech. 591, 145 (2007).

${ }^{12}$ G. Kawahara and S. Kida, "Periodic motion embedded in plane Couette turbulence: Regeneration cycle and burst," J. Fluid Mech. 449, 291 (2001).

${ }^{13}$ O. Flores and J. Jiménez, "Effect of wall-boundary disturbances on turbulent channel flows," J. Fluid Mech. 566, 357 (2006). 